\title{
Flame Symptom Modelling Near Lean Blowout in a Swirl-Stabilized Gas Turbine Model Combustor
}

\author{
Lijun WANG \\ Shenyang Aerospace University \\ Energy and Environment College \\ Shenyang, China \\ wanglijun@sau.edu.cn
}

\author{
Dongdong QI \\ Shenyang Aerospace University \\ Aerospace Engineering Faculty \\ Shenyang, China
}

\author{
Haifeng YANG \\ Shenyang Aerospace University \\ Aerospace Engineering Faculty \\ Shenyang, China
}

\begin{abstract}
Lean premixed combustion offers a practical approach for the reduction of $\mathrm{NO}_{\mathrm{x}}$ emissions, but increases the risk of lean burn blow out (LBO) in gas turbines. Flame behavior in terms of stability is simulated in a model gas turbine combustor burning aviation kerosene at atmospheric pressure. The flame symptom close to LBO, where the flame propagates in the broken-reaction zone (BRZ) regime in double swirl combustor is studied when the main influence parameter of first to second stage air flow ratio is 1:5. Adjusting the dimensionless ratio of oil/air equivalence to 0.35 is identified as significant perturbation source for inducing unstable combustion. Results of simulation show a good agreement with obtained data of other test.
\end{abstract}

Keywords- Turbine lean premixed combustion; Double-stage swirl combustion chamber; Lean blow out Symptom; Numerical simulation.

\section{INTRODUCTION}

For the purpose of reducing NOx emission in gas turbine engines, lean premixed (LP) combustion is adopted as a high effective combustion mode. Highly swirling flows create an internal flow recirculation zone that entrains and circulates a portion of the hot combustion products in the gas turbine combustor dome ${ }^{[1 \sim 3]}$. This recirculation zone not only acts as a heat source, but it plays an active role in better mixing, improving combustion stability, reducing the combustor length and lowing $\mathrm{NO}_{\mathrm{x}}$ emissions. The reaction rates and the flame speed decrease for leaner mixture. If the stabilization method used is not sufficient to sustain the flame inside the combustor, the flame is convected out of the combustor, and this is called blow out of the flame or combustor. The equivalence ratio at which blowout occurs is referred to as the lean blowout (LBO) limit. Flow field analysis can give a crude estimate of the LBO limit for some specified operating condition, but stochastic nature of turbulent flows and perturbations of the operating can move the system beyond the stability of the given combustor $^{[4,5]}$. Currently, stable performance is ensured by operating the combustor with a wide margin above the uncertain LBO limit for multi-swirl stabilized gas turbine combustor, as there is no technology to estimate the proximity to $\mathrm{LBO}^{[6,7]}$.

Recent works have concentrated on understanding the LBO "vortex breakdown" dynamics of bubble mode and the spiral mode in complex flow field using both numerical and experimental methods ${ }^{[8 \sim 9]}$. Two parameters of swirl and the Reynolds number appear to be significantly important to characterize the onset of breakdown in swirling flows. ${ }^{[10 \sim 12]}$

Considering the other influences and authors previous double swirl optimization results of swirl number and air distribution between the double swirl ${ }^{[13]}$, this paper focuses on the flame symptom near lean blowout as fuel/air ratio varied and fixing the conditions of confinement, swirl number and air distribution on the basis of other test. ${ }^{[14]}$

\section{The COMPUTATIONAL MODEL}

A. Physical model and computional grid

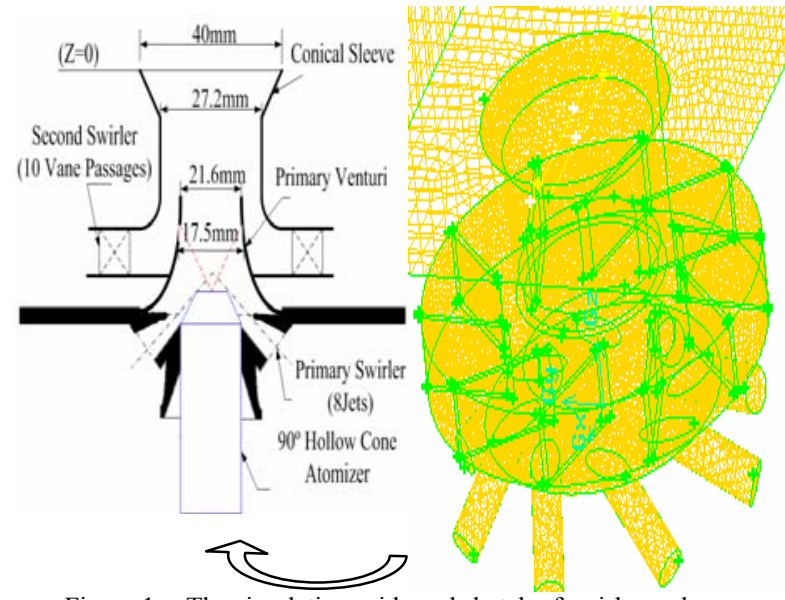

Figure 1. The simulation grids and sketch of swirl cup dome

The numerical model corresponding to the A. M. Elkady's experiment combustor is constructed, as shown in Fig. $1^{[13]}$. The combustor head has installed double swirl cup assembly with a fuel injector. The swirl-cup is considered as a prefilming air blast injector, it consists of eight elliptical tangential primary jets which are used to generate the primary swirling stream and a radial secondary swirler composed of ten curved vanes which introduce the counter-rotating stream into the swirl-cup. The rectangular combustion chamber dimension is $76 \times 76 \times 200 \mathrm{~mm}$. The unstructured mix grid of hexahedral and tetrahedral mesh is used to the double swirl cup configure due to the complex structure. The total number of grids is 520,000 .

Supported by Ministry of Education of Liaoning Province (No.L2010424) 


\section{B. Calculation conditions}

Based on former calculation results of swirl number and air mass inlet distribution, air total flow is $0.035 \mathrm{~kg} / \mathrm{s}$ which of onestage swirl air inlet mass flow is $0.00583 \mathrm{~kg} / \mathrm{s}$ and two-stage swirl air inlet mass flow is $0.02917 \mathrm{~kg} / \mathrm{s}$. Air inlet temperature is $500 \mathrm{~K}$. The test results show that the pressure loss of the combustor was about 4 8\% when burning, and lower when non-burning. The mass ratio (MR) is defined as the ratio of the transverse jets mass flow rate to that of through the swirler, and its value is 0.2 in this calculation case corresponding to A. M. Elkady's test of 0.214 and pressure loss of $5.251 \%$. The calculation cases of (f/a)LBO have not changed MR condition but gradually reduced the fuel mass flow rate near LBO. The parameters at baseline cases are shown in table 1, here, YLBO is dimensionless equivalence ratio of $(\mathrm{f} / \mathrm{a})_{\mathrm{LBO}}$.

TABLE I. CALCULATION PARAMETERS

\begin{tabular}{|c|c|c|c|c|}
\hline $\begin{array}{c}\text { Dimensionless } \\
\text { Equivalence ratio } \\
\boldsymbol{\Psi}_{\text {LBO }}\end{array}$ & 0.6 & 0.5 & 0.4 & 0.35 \\
\hline Fuel supply $\mathrm{kg} / \mathrm{s}$ & 0.001428 & 0.00119 & 0.000952 & 0.000833 \\
\hline
\end{tabular}

\section{The mathematical mode}

Because the vortex zone of the combustor with swirl cup dome and combustion process are very complex, so it is a reasonable and appropriate way to search quantitatively LBO process by using CFD simulation based on the discrete form of $\mathrm{N}-\mathrm{S}$ conservation equations. The governing equations can be written in a generalized form as.

$$
\frac{\partial}{\partial x_{j}}\left(\rho \bar{u}_{i} \phi\right)=\frac{\partial}{\partial x_{j}}\left(\Gamma_{\phi} \frac{\partial \phi}{\partial x_{j}}\right)+S_{\phi}
$$

Here, $\Phi$ denotes common variable of multi-physical such as velocity component $\mathrm{u}, \mathrm{v}, \mathrm{w}$, turbulent kinetic energy $\mathrm{k}$, turbulence dissipation rate $\varepsilon$, f/a mixture fracture $\mathrm{f}$ and its fluctuation $\mathrm{g}$, and temperature $\mathrm{T}$. ГФ represents the diffusive transport coefficient, and $\mathrm{S} \phi$ are the source terms of $\Phi$. The general form of the equation represents conservation of mass, momentum, species, or energy conservation equation et al, depending on the variable used for $\Phi$. The k- $\varepsilon$ turbulence and Non-premixed combustion model PDF are taken. Lagrange method is used to track the movement and transport of the discrete kerosene particles in the whole field, and particles effect on gas flow by added corresponding source term to gas phase conservation equation. In addition, a sink term based on an optically thin gas assumption is included in the energy equation to account for thermal radiation from the flame. P1 radiation and grey model are selected accounts for the absorption and emission from participation gaseous species of which its properties value obtained by using by polynomial approximation provided in FLUENT date base. The standard wall function method is taken to deal with the part near the wall. Total pressure outlet condition is adopted at the combustor outlet boundary, and the inlet flow rate and temperature are also given.

\section{NumERICAL RESUlts NEAR LEAN BLOWOUT AND DISCUSSION}

\section{A. Model validation of non-reacting flow aerodynamic}

Figure 2 presents a comparison between the measured axial velocity contours in the radio-axial plane on the left side and that of computed on the right side for better understanding the flow features of non-reacting flow aerodynamics generated by swirl cup of the same MR. These results confirm the repeatability and the axi-symmetry of the average velocities calculation. The dashed line in the figure represents the zero axial velocity contours which bound the reverse flow region. Due to Coanda effect, the flow departing from the swirler remains attached to the conical sleeve, expands away from the centerline and impinges on the chamber walls. The reverse flow is extended inside the swirl-cup and hence increases the velocity level near the venturi lip. The strong swirling flow induced by the swirler causes high velocity gradients in the immediate vicinity of the swirler and hence high turbulence levels are generated rapidly shown as solid circles. The high swirl motion creates inner recirculation zone (IRZ) that fills outmost of the chamber cross-section in front of the swirler and another outer recirculation zone (ORZ) that ends by zero axial velocity near the chamber walls. Understanding the swirling flow dynamics is important to understand the dynamics of swirl flow with combustion, because the inner recirculation zone(IRZ) is helpful in stabilizing the flame by providing feedback to the incoming gases. In a typical gas turbine combustor, both inner recirculation zone and sudden expansion are used to stabilize the flame at the high combustor loadings required.
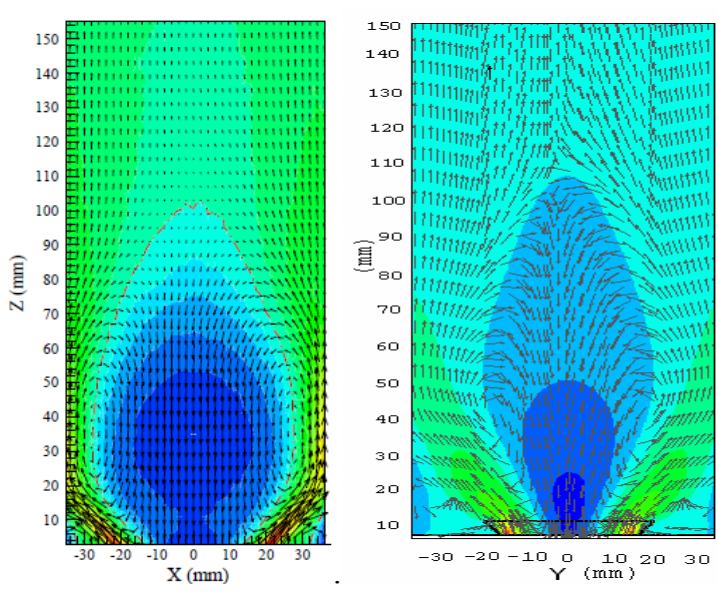

Figure 2. Axial velocity contours of measured and tested

\section{B. Flame symptem near lean blowout}

The variation in combustor operation as LBO approached was numerical studied by holding the overall airflow rate constant and decreasing the fuel flow rate. This ensured that the incoming velocity field was approximately constant while the equivalence ratio was reduced, since the flow rate of the fuel was small compared to that of the air. M. Thiruchengode et $\mathrm{al}^{16]}$ test and discuss the detailed flame structure near blowout of methane-air double swirl cup combustion, Chemiluminescence emission from the $\mathrm{OH}$ radical as flame shape showing was 
recorded over a period of time for various equivalence ratios near blowout, and its result is shown in figure 3 5 on the right side. Figure 3 5 present the computed flame structure in terms of temperature contours on the left side for equivalence ratios of 0.6 to 0.4 .
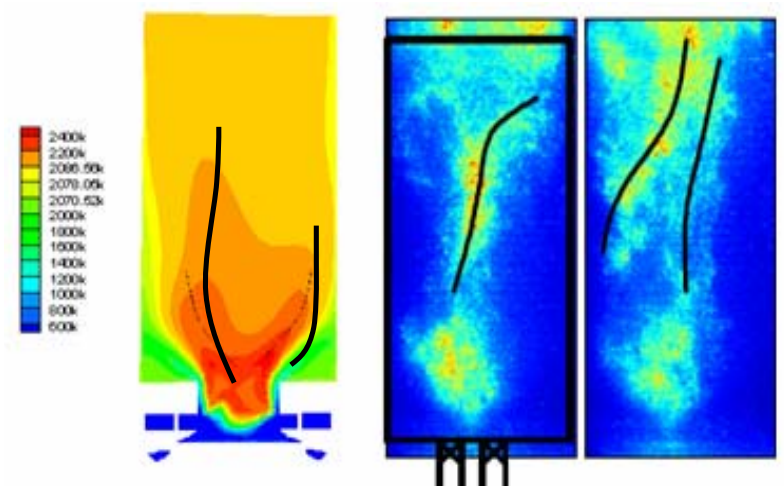

Figure 3. The compression of flame structure near lean blowout for equivalence ratios 0.6

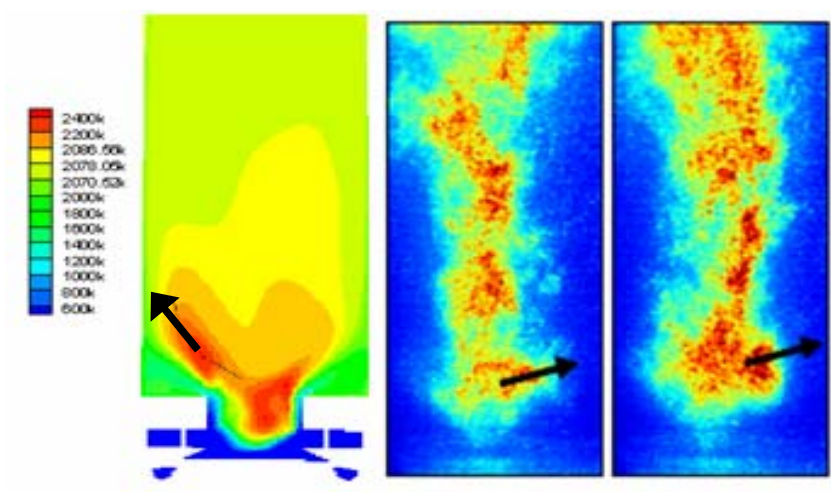

Figure 4. The compression of flame structure near lean blowout for equivalence ratios 0.5

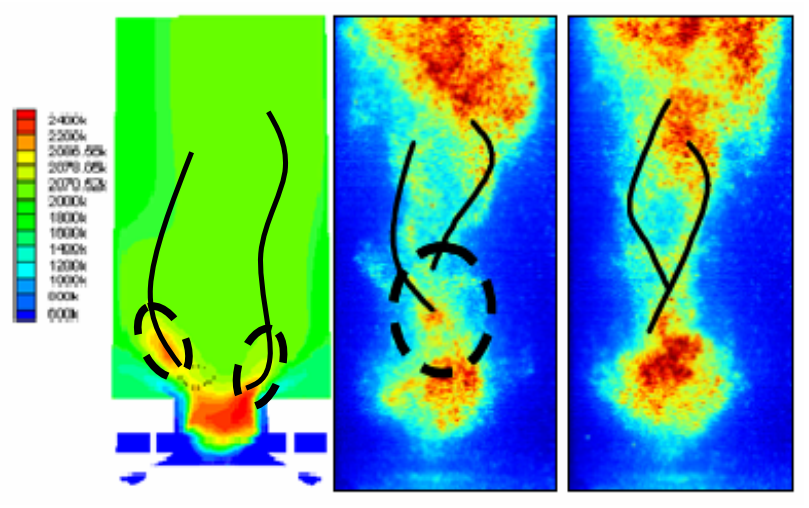

Figure 5. The compression of flame structure near lean blowout for equivalence ratios 0.4

The lines drawn on the flame figures are to facilitate visualizing the movement of flame structures. The circles show the tracking of flame packets (reaction zone around hot/burning gases) as it travels upstream. The arrows show the direction of motion of the flame structure from the current frame to the next frame. It can be found in figure $3 \sim 5$, the flame has the symptom of intermittent behavior near lean blowout for various flames. The flame structure appears to be double helical and the flame packets can be tracked moving downwards. As the equivalence ratio decreasing to 0.35 as depicted in figure 6 , the strength of the flame in the double helix pattern also decreases, and thus the downward flame packets are not sufficient to restore the flame, and the flame blows out.

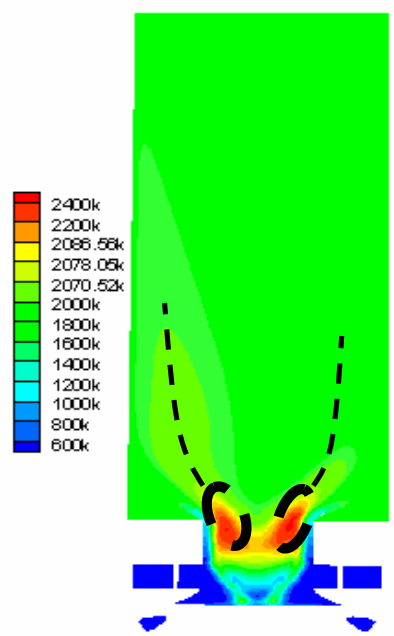

Figure 6. Flame symptoms lean blowout for equivalence ratios 0.35

The differences between these results of computed and tested are that, the liquid fuel is evaporated very quickly due to the hot gas in the IRZ near the inlet. During precursor events, they have the same flame symptoms near flame blowout but gas fueled systems relatively faster mixing contrast to oil fueled which may absorb more heat from hot gas for evaporation. According to the simulation results above, the recirculation region plays the role as the ignition source, which exerts the major influence over whether the fresh combustible mixture could be ignited or not.

Overall, the flame is primarily stabilized by recirculation associated with a (symmetric) bubble-type vortex breakdown. The local extinctions of the flame are likely due to perturbations induced by high strain and low equivalence ratios. The vortex breakdown mode shifts from the bubble type to a spiral/helical mode. The helical mode allows hot products to feedback from farther downstream to the combustor inlet region during the reignition phase of a precursor event. If the feedback is strong enough to cause strong dilatation near the inlet to bring about a shift of the vortex breakdown mode back to a bubble type, the flame is restored in the combustor. If not, the combustor never recovers from the extinction phase and the combustor blows out. This numerical result of lean blowout event is consistent with the behavior of the combustor near blowout as observed in sensing and control experiments. ${ }^{[14]}$

\section{CONCLUSION}

We have presented a computational investigation of the numerical data of flame symptom near lean blowout of a rectangular double swirl cup combustor at atmospheric pressure condition with different fuel/air ratio, while the inlet air distribution and flow velocity remains stable comparatively. The predictions were validated comparing with the experimental analysis of the others, the results are as follows: 
- With the decreasing of the equivalent ratio near blow out, the flame structure shows the double helix structure. The average temperature of the recirculation region will decrease, which is disadvantageous to the ignition of the fresh combustible mixture.

- Flame symptoms near blowout were observed to lose its stability in an intermittent, sporadic and nonsymmetric fashion, and appeared local flameout phenomenon. Continue to reduce the dimensional equivalent $\Psi \mathrm{LBO}$ to 0.35 , the combustor blows out.

- The basic research in this paper can be helpful to understand the process and mechanism of lean blowout in gas turbine combustor, and is beneficial to improve the flame stability performance.

\section{REFERENCES}

[1] Mongia, H. C., "A Fourth Generation Propulsion Combustor Technology for Low Emissions," AIAA International Air and Space Symposium and Exposition: The Next 100 Years, 2003.

[2] Stouffer, S. D., Ballal, D. R., Zelina, J., Shouse, D. T., Hancock, R. D., and Mongia, H. C., "Development and Combustion Performance of a High-Pressure WSR and TAPS Combustor," AIAA pp. 2005-1416, January 2005.

[3] Alejandro M. Briones and Suresh K. Aggarwal, "A numerical investigation of flame liftoff, stabilization, and blowout," PHYSICS OF FLUIDS 18(4), pp. 043603-1 13, 2006
[4] Syred N., Chigier N.A., and Beer J.M., "Flame stabilization in recirculation zones of jets with swirl," Proc. Comb. Inst., vol. 13, pp. 617-624, 1971

[5] Kurosoy E., and Whitelaw J. H., "Extinction and relight in opposed flames," Experiments in Fluids, Vol. 33, pp. 75-89, 2002

[6] James F. Driscoll, Chadwick C. Rasmussen, "Correlation and analysis of blowout limits of flames in high-speed airflows," Journal of propulsion and power, Vol. 21, No.6, pp. 1034-1044, 2005

[7] Richard J. Roby, Michael S. Klassen, Leo D. Eskin, "Development of a system for lean, prevaporized, premixed combustion," Proceedings of the 34rd. Turbomachinery Sysposium, pp. 55-60, 2007

[8] M. Stohr, I. Boxx, C. Carter, et al, "Dynamics of lean blowout of a swirlstabilized flame in a gas turbine model combustor," Proceedings of the Combustion Institute, vol. 33. pp. 2953-2960, 2011

[9] YiXiang Yuan, Hailin Fan, Xiyang Liu, et al, "Analysis on lean blowout of swirl cup combustor at atmospheric pressure condition," Vol.4, No.4 pp. 349-354, 2011

[10] M. P. Escudier, N. Zehnder, "Vortex-Flow regimes (Vortex breakdown criterion)," Vol. 115, pp. 105-121, 1982

[11] M. P. Escudier, J. J. Keller, "Recirculation in swirling flow: A manifestation of vortex breakdown," AIAA, Journal, Vol. 23, No.1, pp 111-116, 1985

[12] C. Brucker, R. Althaus, "Study of vortex breakdown by particle tracking velocimetry (PIV), Part 1: Bubble-type vortex breakdown," Experiments in Fluids, Vol. 13, pp. 339-349, 1992

[13] Ahmed M. ELKady, "Experimental Investigation of Aerodynamics, Combustion, and Emissions Characteristics within the Primary Zone of a Gas Turbine Combustor, ” pp. 28-48, 2005

[14] T. M. Muruganandam, S. Nair, D. Scarborough, "Active Control of Lean Blowout for Turbine Engine Combustors, Journal of propulsion and power," Vol. 21, No.5, pp. 807-814, 2005. 